\title{
Investigating the Functional Role of Callosal Connections with Dynamic Causal Models
}

\author{
KLAAS E. STEPHAN, ${ }^{a}$ WILL D. PENNY, ${ }^{a}$ JOHN C. MARSHALL, ${ }^{b}$ \\ GEREON R. FINK, ${ }^{c, d}$ AND KARL J. FRISTON ${ }^{a}$ \\ ${ }^{a}$ Wellcome Department of Imaging Neuroscience, Institute of Neurology, \\ University College London, London WC1N 3BG, United Kingdom \\ ${ }^{b}$ Neuropsychology Unit, University Department of Clinical Neurology, \\ Radcliffe Infirmary, Oxford OX2 6HE, United Kingdom \\ ${ }^{c}$ Institute of Medicine (IME), Research Center Jülich, 52425 Jülich, Germany \\ ${ }^{d}$ Department of Neurology/Cognitive Neurology, University of Aachen, \\ 52074 Aachen, Germany
}

\begin{abstract}
The anatomy of the corpus callosum has been described in considerable detail. Tracing studies in animals and human postmortem experiments are currently complemented by diffusion-weighted imaging, which enables noninvasive investigations of callosal connectivity to be conducted. In contrast to the wealth of anatomical data, little is known about the principles by which interhemispheric integration is mediated by callosal connections. Most importantly, we lack insights into the mechanisms that determine the functional role of callosal connections in a context-dependent fashion. These mechanisms can now be disclosed by models of effective connectivity that explain neuroimaging data from paradigms that manipulate interhemispheric interactions. In this article, we demonstrate that dynamic causal modeling (DCM), in conjunction with Bayesian model selection (BMS), is a powerful approach to disentangling the various factors that determine the functional role of callosal connections. We first review the theoretical foundations of DCM and BMS before demonstrating the application of these techniques to empirical data from a single subject.
\end{abstract}

KEYWORDS: fMRI; DTI; DCM; effective connectivity; corpus callosum; interhemispheric integration

\section{INTRODUCTION}

Ever since the description of localizable lesion and excitation effects in the nineteenth century, modern neuroscience has revolved around the twin themes of functional specialization and functional integration. ${ }^{1,2}$ Functional specialization refers to the notion that local neural units are specialized in certain aspects of information processing, for example, the processing of particular stimulus properties. Traditional

Address for correspondence: Klaas E. Stephan, Wellcome Department of Imaging Neuroscience, Institute of Neurology, University College London, 12 Queen Square, London WC1N 3BG, United Kingdom. Voice: +44-207-8337481; fax: +44-207-8131420.

k.stephan@fil.ion.ucl.ac.uk

Ann. N.Y. Acad. Sci. 1064: 16-36 (2005). (C) 2005 New York Academy of Sciences. doi: 10.1196/annals.1340.008 
methods for investigating functional specialization include invasive recordings from animals and neuropsychological investigations of patients with brain lesions. More recently, functional neuroimaging techniques like functional magnetic resonance imaging (fMRI) have made it possible to investigate functional specialization across the whole brain in a noninvasive manner. In contrast, functional integration refers to the causal interactions among distinct neural units. Critically, the form of these causal interactions, which mediate complex cognitive processes, is constrained by the anatomical connections between the neural units. Consequently, in order to understand the basis of functional integration, much effort has been invested in characterizing anatomical connectivity in the mammalian brain. For example, thousands of tract tracing experiments have been performed in several species over the last decades. These techniques require the in vivo injection of specific dyes into particular areas. Depending on its biophysical properties, the dye is taken up by neuronal somata or axonal terminals and transported in an anterograde or retrograde direction, respectively. ${ }^{3}$ Subsequent histological processing of the brain can then reveal the regions that receive connections from (anterograde tracer) or send connections to (retrograde tracer) the injected area. For the macaque monkey alone, more than 36,000 individual experimental findings from tracing experiments are described by the connectivity database $\mathrm{CoCoMac}^{4}$ (see www.cocomac.org).

Unfortunately, for the human brain, we have considerably less knowledge about its anatomical connections. This is because tract tracing procedures, as the goldstandard method to reveal anatomical connections, are too invasive to use in the human brain. There has been an intensive search for postmortem methods as an alternative to investigate human brain connectivity, but these methods are either restricted to very large fiber bundles ${ }^{5}$ or limited to very short intracortical connections. ${ }^{6}$

Recently, the advent of noninvasive diffusion-weighted imaging (DWI) techniques, for example, diffusion tensor imaging (DTI), has raised great hopes that we may be able to obtain a complete picture of human brain connectivity in the not too distant future. However, the resolution of current DWI approaches is still too coarse to allow for characterizations that are comparable to those obtained from tracing techniques, and fundamental problems like intravoxel fiber crossings still need to be solved convincingly. Using improved acquisition schemes, probabilistic approaches, ${ }^{7,8}$ and models that do not make strong a priori assumptions about the shape of the spatial distribution of diffusion, ${ }^{9}$ these technical limitations might eventually be overcome. Should this indeed be possible, would this mean that we have all the information we need to understand principles of functional integration in the human brain? The answer is, unfortunately, no. Even if we knew everything about the anatomical connectivity of a particular neural system, we cannot directly derive its dynamics from its connectional structure. ${ }^{10}$ Further knowledge is required, for example, of the time constants of activity propagation or the strength of individual connections and how these change as a function of cognitive context (task requirements, learning, etc.). ${ }^{11}$ These time constants and connection strengths are parameters that have to be estimated from empirical observations. In conclusion, knowledge of anatomical connectivity is a necessary, but not sufficient, condition to build dynamical models of brain function.

One good example is the corpus callosum. This massive fiber bundle, which contains a huge number of axons that link and functionally integrate the two hemispheres, has been investigated in great detail. We know the source and target laminae 
of neurons projecting through the corpus callosum, ${ }^{12}$ the spatial distribution of axonal diameters across the callosum, ${ }^{13}$ and the topography of individual callosal projections. ${ }^{14,15}$ Neuropsychological studies have demonstrated the involvement of very restricted parts of the corpus callosum in specific cognitive processes. ${ }^{16}$ More recently, the corpus callosum has been studied intensively by DWI studies that have investigated its connectivity in both healthy subjects ${ }^{17}$ and patients. ${ }^{18}$ Yet, in spite of all these data, we still lack any powerful theory of callosal function and how it underlies interhemispheric integration. Banich and colleagues have recently advanced a useful framework that relates the functional role of the corpus callosum to the complexity of cognitive tasks and attentional processing, ${ }^{19}$ but this theory is neither quantitative nor directly embedded into a precise neurobiological model.

An important starting point for more precise theories of callosal function would be to investigate neurobiologically plausible models of interhemispheric integration in order to (i) identify those cognitive factors that determine the functional role of specific callosal connections, (ii) determine quantitatively the strength of callosal connections as a function of experimentally controlled cognitive context, and (iii) analyze the pattern of context-dependent connection strengths with regard to interesting features, for example, directional asymmetries or differences in modulation by different cognitive factors. Models of effective connectivity, which are based on empirical neuroimaging data and model the modulation of connection strengths by experimentally controlled changes in context, would be ideal to do this. Previously available techniques for studying effective connectivity, for example, structural equation modeling (SEM), are not well suited for models of high connectional complexity, for example, multiple reciprocal connections and loops, due to potential problems of identifiability. ${ }^{20}$ This is a particular problem for models of interhemispheric integration because callosal connections appear to be generally reciprocal between homotopic regions. ${ }^{21,22}$ Furthermore, SEM operates at the level of measured hemodynamic responses and does not offer a model of the underlying neural processes.

In this article, we demonstrate how a novel method to study effective connectivity, dynamic causal modeling $\left(\mathrm{DCM}^{11}\right)$, can be combined with Bayesian model selection $\left(\mathrm{BMS}^{23}\right.$ ) to address quite complex questions about callosal function. First, we briefly review the theoretical foundations of both DCM and BMS. Subsequently, we apply DCM to fMRI data from a single subject who performed a task that manipulated interhemispheric interactions. Specifically, we focus on the question of how competing hypotheses about the functional role of callosal connections in a specific cognitive context can be disambiguated using BMS.

\section{METHODS}

\section{Dynamic Causal Modeling (DCM)}

DCM is a method to make inferences about neural processes that underlie measured time series-in our case, fMRI data. The general idea is to estimate the parameters of a reasonably realistic neuronal system model such that the predicted blood oxygen level-dependent (BOLD) signal, which results from converting the modeled neural dynamics into hemodynamic responses, corresponds as closely as possible to the 


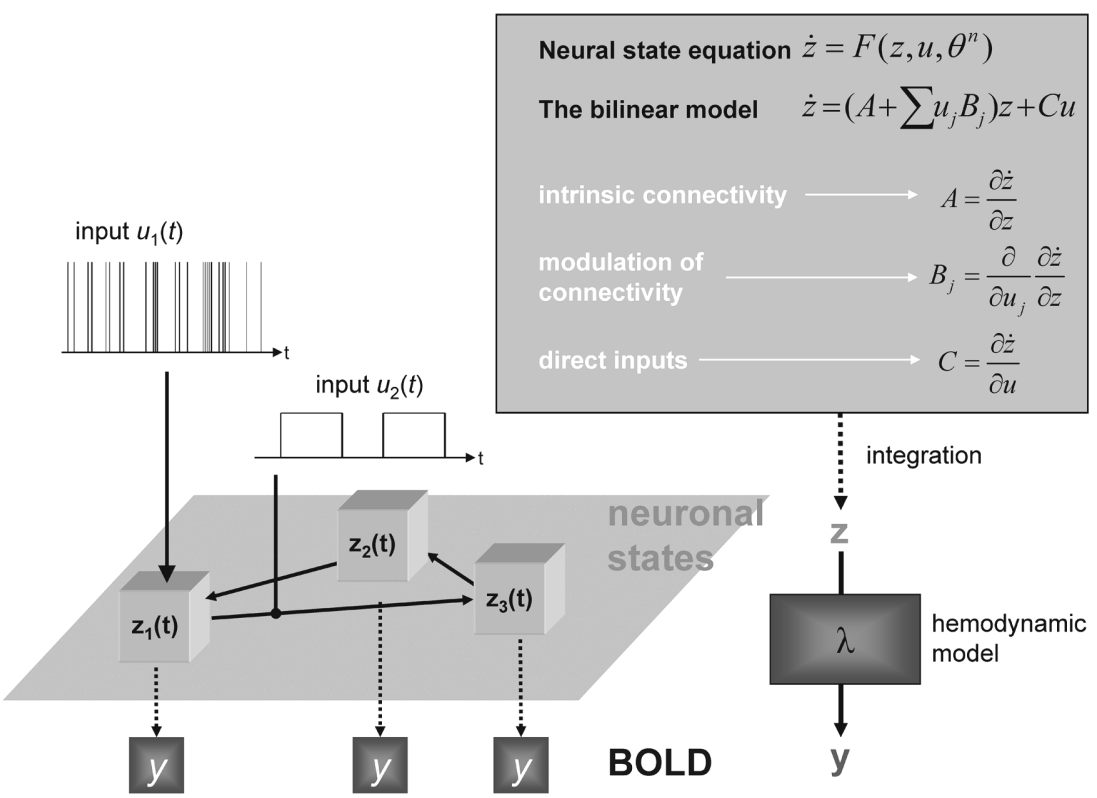

FIGURE 1. Schematic summary of the conceptual basis of DCM. The dynamics in a system of interacting neuronal populations (left lower panel), which are not directly observable by fMRI, is modeled using a bilinear state equation (right upper panel). Integrating the state equation gives predicted neural dynamics $(z)$ that enter a model of the hemodynamic response $(\lambda)$ to give predicted BOLD responses $(y)$ (right lower panel). The parameters at both neural and hemodynamic levels are adjusted such that the differences between predicted and measured BOLD series are minimized. Critically, the neural dynamics are determined by experimental manipulations. These enter the model in the form of external inputs (left upper panel). Driving inputs $\left(u_{1}\right.$; e.g., sensory stimuli) elicit local responses directly that are propagated through the system according to the intrinsic connections. The strengths of these connections can be changed by modulatory inputs $\left(u_{2}\right.$; e.g., changes in cognitive set, attention, or learning). In this figure, the structure of the system and the scaling of the inputs are arbitrary.

observed BOLD time series. As in state-space models, two distinct levels constitute a DCM (see FIG. 1). The hidden level, which cannot be directly observed using fMRI, represents a simple model of neural dynamics in a system of $k$ coupled brain regions. Each system element $i$ is represented by a single state variable $z_{i}$, and the dynamics of the system is described by the change of the neural state vector $z=\left[z_{1}, \ldots, z_{k}\right]^{T}$ over time. The neural state variables do not correspond directly to any common neurophysiological measurement (such as spiking rates or local field potentials), but represent a summary index of neural population dynamics in the respective regions. Importantly, DCM models how the neural dynamics are driven by external perturbations that result from experimentally controlled manipulations. These perturbations are described by means of external inputs $u$ that enter the model in two different ways: they can elicit responses through direct influences on specific regions ("driving" inputs, e.g., evoked responses in early sensory areas) or they can change 
the strength of coupling among regions ("modulatory" inputs, e.g., during learning or attention). Overall, DCM models the temporal evolution of the neural state vector, that is, $\dot{z}=d z / d t$, as a function of the current state, the inputs $u$, and some parameters $\theta^{n}$ that define the functional architecture and interactions among brain regions at a neuronal level ( $n$ is not an exponent, but simply denotes "neural"):

$$
\left[\begin{array}{lll}
\dot{z}_{1} & \ldots & \dot{z}_{k}
\end{array}\right]=\dot{z}=F\left(z, u, \theta^{n}\right)
$$

In this neural state equation, the state $z$ and the inputs $u$ are time-dependent, whereas the parameters are time-invariant. In DCM, $F$ has the bilinear form

$$
\dot{z}=A z+\sum_{j=1}^{m} u_{j} B_{j} z+C u \text {. }
$$

The parameters of this bilinear neural state equation, $\theta^{n}=\left\{A, B_{1}, \ldots, B_{m}, C\right\}$, can be expressed as partial derivatives of $F$ :

$$
\begin{gathered}
A=\partial F / \partial z=\partial \dot{z} / \partial z \\
B_{j}=\partial^{2} F / \partial z \partial u_{j}=\left(\partial / \partial u_{j}\right)(\partial \dot{z} / \partial z) \\
C=\partial F / \partial u .
\end{gathered}
$$

These parameter matrices describe the nature of the three causal components that underlie the modeled neural dynamics: (i) context-independent effective connectivity among brain regions, mediated by anatomical connections $(k \times k$ matrix $A)$; (ii) contextdependent changes in effective connectivity induced by the $j$-th input $u_{j}(k \times k$ matrices $\left.B_{1}, \ldots, B_{m}\right)$; and (iii) direct inputs into the system that drive regional activity $(k \times m$ matrix $C$ ). As will be demonstrated below, the posterior distributions of these parameters can inform us about the impact that different mechanisms have on determining the dynamics of the model. Notably, the distinction between "driving" and "modulatory" is neurobiologically relevant: driving inputs exert their effects through direct synaptic responses in the target area, whereas modulatory inputs change synaptic responses in the target area in response to inputs from another area. This distinction represents an analogy, at the level of large neural populations, to the concept of driving and modulatory afferents in studies of single neurons. ${ }^{24}$

DCM combines this model of neural dynamics with a biophysically plausible and experimentally validated hemodynamic model that describes the transformation of neuronal activity into a BOLD response. This so-called "balloon model" was initially formulated by Buxton and colleagues ${ }^{25}$ and later extended by Friston et al. ${ }^{26,27}$ Briefly summarized, it consists of a set of differential equations that describe the relations between four hemodynamic state variables, using five parameters $\left(\theta^{h}\right)$. More specifically, changes in neural activity elicit a vasodilatory signal that leads to increases in blood flow and subsequently to changes in blood volume $v$ and deoxyhemoglobin content $q$. The predicted BOLD signal $y$ is a nonlinear function of blood volume and deoxyhemoglobin content: $y=\lambda(v, q)$. Details of the hemodynamic model can be found in other publications. ${ }^{11,26,27}$ 
By combining the neural and hemodynamic states into a joint state vector $x$ and the neural and hemodynamic parameters into a joint parameter vector $\theta=\left[\theta^{n} \theta^{h}\right]^{T}$, we obtain the full forward model that is defined by the neural and hemodynamic state equations

$$
\begin{gathered}
\dot{x}=F(x, u, \theta) \\
y=\lambda(x) .
\end{gathered}
$$

For any given set of parameters $\theta$ and inputs $u$, the joint state equation can be integrated and passed through the output nonlinearity $\lambda$ to give a predicted BOLD response $h(u, \theta)$. This can be extended to an observation model that includes observation error $\varepsilon$ and confounding effects $X$ (e.g., scanner-related low-frequency drifts):

$$
y=h(u, \theta)+X \beta+\varepsilon .
$$

This formulation is the basis for estimating the neural and hemodynamic parameters from the measured BOLD data, using a fully Bayesian approach with empirical priors for the hemodynamic parameters and conservative shrinkage priors for the neural coupling parameters. Details of the parameter estimation scheme, which rests on a Gauss-Newton gradient ascent embedded in an expectation maximization (EM) algorithm, can be found elsewhere. ${ }^{11}$ In brief, under Gaussian assumptions about the posterior distributions (Laplace approximation), this scheme returns the posterior expectations ( $=$ maximum a posteriori [MAP] estimates) $\eta_{\theta \mid y}$ and posterior covariance $C_{\theta \mid y}$ for the parameters as well as hyperparameters for the covariance of the observation noise, $C_{\varepsilon}$.

After fitting the model to measured BOLD data, the posterior distributions of the parameters can be used to test hypotheses about the size and nature of effects at the neural level. Although inferences could be made about any of the parameters in the model, hypothesis testing usually concerns context-dependent changes in coupling (i.e., specific parameters from the $B$ matrices). As will be demonstrated below, at the single-subject level, these inferences concern the question of how certain one can be that a particular parameter or, more generally, a contrast of parameters, $c^{T} \eta_{\theta \mid y}$, exceeds a particular threshold $\gamma$ (e.g., zero; see FIG. 6 later). Under the assumptions of the Laplace approximation, this is easy to test $\left(\phi_{N}\right.$ denotes the cumulative normal distribution):

$$
p\left(c^{T} \eta_{\theta \mid y}>\gamma\right)=\phi_{N}\left[\left(c^{T} \eta_{\theta \mid y}-\gamma\right) /\left(\sqrt{c^{T} C_{\theta \mid y} c}\right)\right] .
$$

For example, for the special case $c^{T} \eta_{\theta \mid y}=\gamma$, the probability is $p\left(c^{T} \eta_{\theta \mid y}>\gamma\right)=50 \%$, that is, it is equally likely that the parameter is smaller or larger than the chosen threshold $\gamma$.

We conclude this section on the theoretical foundations of DCM by noting that the parameters can be understood as rate constants (units: $1 / \mathrm{s}=\mathrm{Hz}$ ) of neural population responses that have an exponential nature (see also figure 1 in ref. 28). This is easily understood if one considers that the solution to a linear ordinary differential equation of the form $\dot{z}=A z$ is an exponential function (compare the state equation in equation 2). 


\section{Bayesian Model Selection (BMS)}

A generic problem encountered by any kind of modeling approach is the question of model selection: given some observed data, which of several alternative models is the optimal one? This problem is not trivial because the decision cannot be made solely by comparing the relative fit of the competing models. One also needs to take into account the relative complexity of the models as expressed, for example, by the number of free parameters in each model. Model complexity is important to consider because there is a trade-off between model fit and generalizability (i.e., how well the model explains different data sets that were all generated from the same underlying process). As the number of free parameters is increased, model fit increases monotonically, whereas beyond a certain point model generalizability decreases. The reason for this is "overfitting": an increasingly complex model will, at some point, start to fit noise that is specific to one data set and thus become less generalizable across multiple realizations of the same underlying generative process. (Generally, in addition to the number of free parameters, the complexity of a model also depends on its functional form. ${ }^{29}$ This is not an issue for DCM, however, because here all possible models have the same functional form.)

Therefore, the question, "what is the optimal model?", can be reformulated more precisely as "what is the model that represents the best balance between fit and complexity?". In a Bayesian context, the latter question can be addressed by comparing the evidence, $p(y \mid m)$, of different models. According to Bayes theorem

$$
p(\theta \mid y, m)=[p(y \mid \theta, m) p(\theta \mid m)] / p(y \mid m),
$$

the model evidence can be considered as a normalization constant for the product of the likelihood of the data and the prior probability of the parameters; therefore,

$$
p(y \mid m)=\int p(y \mid \theta, m) p(\theta \mid m) d \theta .
$$

Here, the number of free parameters (as well as the functional form) are considered by the integration. Unfortunately, this integral cannot usually be solved analytically; therefore, an approximation to the model evidence is needed.

In the context of DCM, one potential solution could be to make use of the Laplace approximation, that is, to approximate the model evidence by a Gaussian that is centered on its mode. As shown by Penny et al. ${ }^{23}$ this yields the following expression for the natural logarithm $(\mathrm{ln})$ of the model evidence $\left(\eta_{\theta \mid y}\right.$ denotes the MAP estimate, $C_{\theta \mid y}$ is the posterior covariance of the parameters, $C_{\varepsilon}$ is the error covariance, $\theta_{p}$ is the prior mean of the parameters, and $C_{p}$ is the prior covariance):

$$
\begin{aligned}
\ln p(y \mid m) & =\operatorname{accuracy}(m)-\operatorname{complexity}(m) \\
& =\left\{(-1 / 2) \ln \left|C_{\varepsilon}\right|-(1 / 2)\left[y-h\left(u, \eta_{\theta \mid y}\right)\right]^{T} C_{\varepsilon}{ }^{-1}\left[y-h\left(u, \eta_{\theta \mid y}\right)\right]\right\}- \\
& {\left[(1 / 2) \ln \left|C_{p}\right|-(1 / 2) \ln \left|C_{\theta \mid y}\right|+(1 / 2)\left(\eta_{\theta \mid y}-\theta_{p}\right)^{T} C_{p}{ }^{-1}\left(\eta_{\theta \mid y}-\theta_{p}\right)\right] . }
\end{aligned}
$$

This expression properly reflects the requirement, as discussed above, that the optimal model should represent the best compromise between model fit (accuracy) and model complexity. The complexity term depends on the prior density, for example, the prior covariance of the intrinsic connections (see eq. 9). This is problematic in the context of DCM for fMRI because this prior covariance is defined in a model- 
specific fashion to ensure that the probability of obtaining an unstable system is very small. (Specifically, this is achieved by choosing the prior covariance of the intrinsic coupling matrix $A$ such that the probability of obtaining a positive Lyapunov exponent of $A$ is $P<0.001$; see Friston et al. ${ }^{11}$ for details.) Consequently, one cannot easily compare models with different numbers of connections. Therefore, alternative approximations to the model evidence are useful for DCMs of this sort.

Suitable approximations, which do not depend on the prior density, are afforded by the Bayesian information criterion (BIC) and Akaike information criterion (AIC), respectively. As shown by Penny et al. ${ }^{23}$ these approximations for DCM are given by

$$
\begin{gathered}
\mathrm{BIC}=\operatorname{accuracy}(m)-\left(d_{\theta} / 2\right) \ln N \\
\mathrm{AIC}=\operatorname{accuracy}(m)-d_{\theta},
\end{gathered}
$$

where $d_{\theta}$ is the number of parameters and $N$ is the number of data points (scans). If one compares the complexity terms of BIC and AIC, it becomes obvious that BIC pays a heavier penalty than AIC as soon as one deals with 8 or more scans (which is virtually always the case for fMRI data):

$$
\left(d_{\theta} / 2\right) \ln N>d_{\theta} \Rightarrow N>e^{2} \approx 7.39
$$

Therefore, BIC will be biased towards simpler models, whereas AIC will be biased towards more complex models. This can lead to disagreement between the two approximations about which model should be favored. We have thus adopted the convention that, for any pairs of models $m_{i}$ and $m_{j}$ to be compared, a decision is only made if AIC and BIC concur; the decision is then based on that approximation that gives the smaller Bayes factor $(\mathrm{BF})$ :

$$
\mathrm{BF}_{i j}=p\left(y \mid m_{i}\right) / p\left(y \mid m_{j}\right) .
$$

This approach to BMS is a robust procedure to decide between competing hypotheses represented by different DCMs. These hypotheses can concern any part of the structure of the modeled system, for example, the pattern of intrinsic connections or which inputs affect the system and where they enter. Below, we will show a concrete example that demonstrates how the combination of DCM and BMS can be applied in practice to disclose previously unknown principles of interhemispheric integration.

\section{Some Considerations on the Study of Interhemispheric Integration}

Interhemispheric integration appears to be an ongoing process that is invoked by any cognitive task and cannot be abolished voluntarily. The question for the experimentalist is therefore not how to induce or prevent interhemispheric integration, but rather how to alter the form it takes. Two experimental manipulations are particularly effective for so doing. First, there are various ways of delivering sensory stimuli such that one hemisphere is initially or preferentially affected by these stimuli. For example, due to the topography of the anatomical connections from the retina to the visual cortex, presentation of visual stimuli in the periphery of one visual hemifield ensures that the contralateral visual cortex receives the stimulus information first. Therefore, 
one knows that any area that is in the hemisphere ipsilateral to stimulus presentation can only receive this information if it is transferred through the corpus callosum (or some alternative, e.g., subcortical, commissure). Second, if one uses a strongly lateralized task that draws on easily identified areas, one knows the target hemisphere and the target areas that require the stimulus information.

Combining both approaches allows one to predict precisely where the stimulus information initially enters the system and to which areas of the system it must be transferred. The challenge is then to characterize the potential paths of information flow and how these pathways are modulated by cognitive context. Rephrasing the question about the nature of interhemispheric integration in this way shows that we are dealing with a generic system identification problem that, in the context of neuroimaging, is best addressed using models of effective connectivity. ${ }^{1,30}$ Unfortunately, very few neuroimaging studies have been conducted so far that directly tackle the question of interhemispheric integration on the basis of a precisely defined system model (see McIntosh et al. ${ }^{31}$ for an exception).

In the next sections, we will provide an example for using DCM and BMS to address the mechanisms underlying interhemispheric integration. This example focuses on the ventral stream of the visual system and is motivated by a recent study that combined the two experimental manipulations described above, that is, using two strongly and inversely lateralized tasks operating on identical visual stimuli that were presented peripherally in the visual hemifields. ${ }^{32}$

\section{Interhemispheric Integration in the Ventral Stream of the Visual System}

In a previous fMRI study on the mechanisms underlying hemispheric specialization, we investigated whether lateralization of brain activity depends on the nature of the sensory stimuli or on the nature of the cognitive task performed. ${ }^{32}$ For example, microstructural differences between homotopic areas in the left and right hemisphere have been reported, including visual ${ }^{33}$ and language-related ${ }^{34}$ areas. Within a given hemisphere, these differences could favor the processing of certain stimulus characteristics and disadvantage others and might thus support stimulus-dependent lateralization in a bottom-up fashion. ${ }^{35}$ On the other hand, processing demands, mediated through cognitive control processes, might determine in a top-down fashion which hemisphere obtains precedence in a particular task context. ${ }^{36,37}$ To decide between these two possibilities, we used a paradigm in which the stimuli were kept constant throughout the experiment, and subjects were alternately instructed to attend to certain stimulus features and ignore others. ${ }^{32}$ The stimuli were concrete German nouns (each four letters in length) in which either the second or third letter was printed in red (the other letters were black). In a letter decision (LD) task, the subjects had to ignore the position of the red letter and indicate whether or not the word contained the target letter "A". In a spatial decision (SD) task, they were required to ignore the language-related properties of the word and to judge whether the red letter was located left or right of the word center: $50 \%$ of the stimuli were presented in the nonfoveal part of the right visual field (RVF) and the other $50 \%$ in the nonfoveal part of the left visual field (LVF).

The results of a conventional fMRI data analysis were clearly in favor of the topdown hypothesis: despite the use of identical word stimuli in all conditions, comparing spatial to letter decisions showed strongly right-lateralized activity in the parietal 


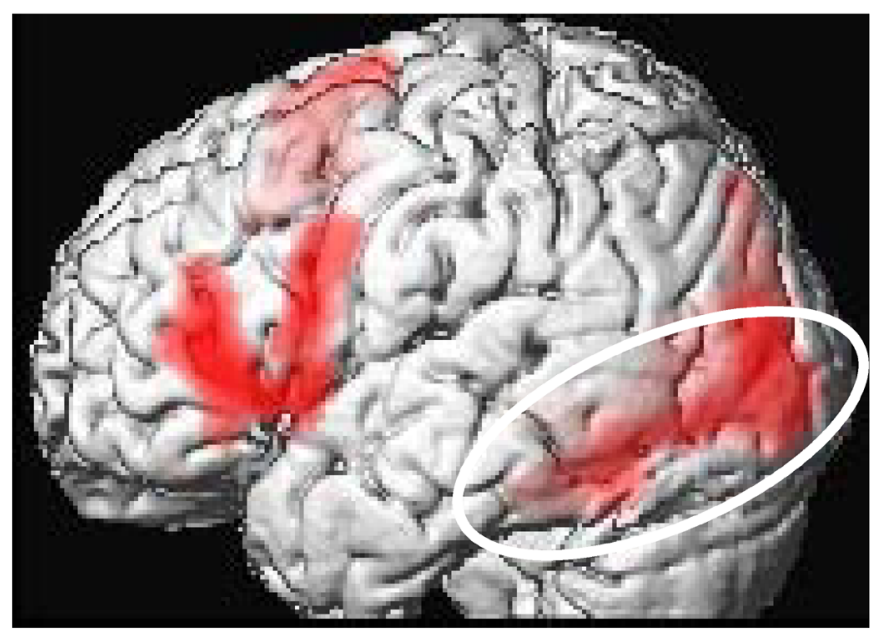

\section{letter decisions > spatial decisions}

FIGURE 2. Results from a conventional analysis of the fMRI data by Stephan et al. ${ }^{32}$ using SPM99. Comparing letter decisions to visuospatial decisions about identical stimuli showed strongly left-lateralized activity, including classical language areas in the left inferior frontal gyrus and visual areas in the left ventral visual stream (white ellipse), for example, in the fusiform gyrus, middle occipital gyrus, and lingual gyrus. Results are shown at $P<0.05$, corrected at the cluster level for multiple comparisons across the whole brain. Adapted with permission from figure 1 in reference 32 .

cortex, whereas comparing letter to visuospatial decisions showed strongly leftlateralized activity, including classical language areas in the left inferior frontal gyrus and visual areas in the left ventral visual stream, for example, in the fusiform gyrus (FG), middle occipital gyrus (MOG), and lingual gyrus (LG) (see FIG. 2).

We now want to demonstrate how one can use DCM to investigate interhemispheric interactions with this paradigm. We focus on the ventral stream of the visual system, which, as shown in FIGURE 2, is preferentially involved in letter decisions in this experiment. For simplicity, we initially omit MOG and concentrate on LG and FG. First, we need to define a model comprising these four areas (FIG. 3A). To start with the direct (driving) inputs to the system, we model the lateral stimulus presentation and the crossed course of the visual pathways by allowing all RVF stimuli to directly affect left LG activity and all LVF stimuli to directly affect right LG activity, regardless of task. Each stimulus lasted for $150 \mathrm{~ms}$ only; therefore, these inputs are represented as trains of short events (delta functions). The induced activity then spreads through the system according to the intrinsic connections of the model. For visual areas, it is biologically plausible to assume that both the intra- and the interhemispheric connections are reciprocal and that homotopic regions in both hemispheres are linked by interhemispheric connections. ${ }^{14,21,22,38,39}$

Note that up to this point there are few, if any, plausible alternatives for how a DCM of interhemispheric integration between LG and FG, respectively, should be constructed. The important question, however, is how transcallosal information 
A

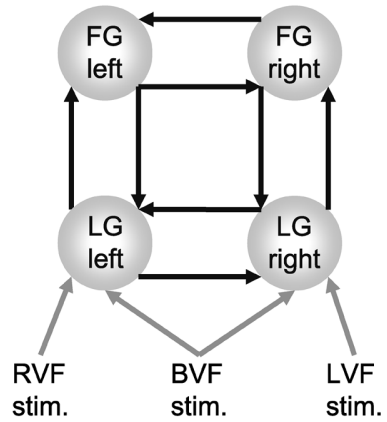

B

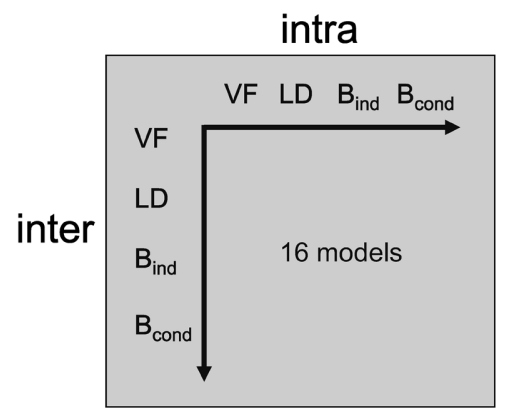

FIGURE 3. (A) Basic structure of a model that comprises the left and right lingual gyrus (LG) and left and right fusiform gyrus (FG). The areas are reciprocally connected (black arrows). Driving inputs are shown as gray arrows. RVF stimuli directly affect left LG activity and LVF stimuli directly affect right LG activity, regardless of task context. Individual stimuli lasted for $150 \mathrm{~ms}$ only; therefore, these inputs are represented as trains of events (delta functions). During the instruction periods, bilateral visual field input was provided for $6 \mathrm{~s}$; this was modeled as a box-car input affecting LG in both hemispheres. (B) Schema showing how the 16 models tested were constructed by systematically combining four different types of modulatory inputs for inter- and intrahemispheric connections; these different inputs are described by FIGURE 4.

transfer is regulated by cognitive set. For example, one could assume that the strengths of interhemispheric interactions between visual areas are merely determined by the visual field of stimulus presentation, regardless of what the subject is instructed to do with the stimulus: whenever a stimulus is presented in the LVF, for example, and stimulus information is thus received initially by the right visual cortex, this information is transmitted transcallosally to the left visual cortex. Vice versa, whenever a stimulus is presented in the RVF, stimulus information is transmitted transcallosally from left to right visual cortex. In this scenario, the task performed is assumed to have no influence on callosal couplings. In contrast to this notion, results from previous analyses of these data by simple models of effective connectivity have indicated the importance of task demands on modulating functional couplings within hemispheres. ${ }^{32}$ If this finding extends to interhemispheric interactions, one might expect that callosal connection strengths depend more on which task is performed than on which visual field the stimulus is presented in. That is, both right $\rightarrow$ left and left $\rightarrow$ right callosal connections could be enhanced during the LD task, enabling a tight cooperation of the two hemispheres during the task. As a third hypothesis, it is conceivable that both visual field and task exert an influence on callosal connection strengths, but independently of each other. As a fourth and final option, one might postulate that task demands modulate callosal connections, but are conditional on the visual field, that is, right $\rightarrow$ left connections are only modulated by LD during LVF stimulus presentation (LD $\mid \mathrm{LVF})$, whereas left $\rightarrow$ right connections are only modulated by LD during RVF stimulus presentation (LD|RVF).

Each of these hypotheses of how cognitive set may modulate the callosal connections represents a different DCM, describing the mechanisms that caused the observed 


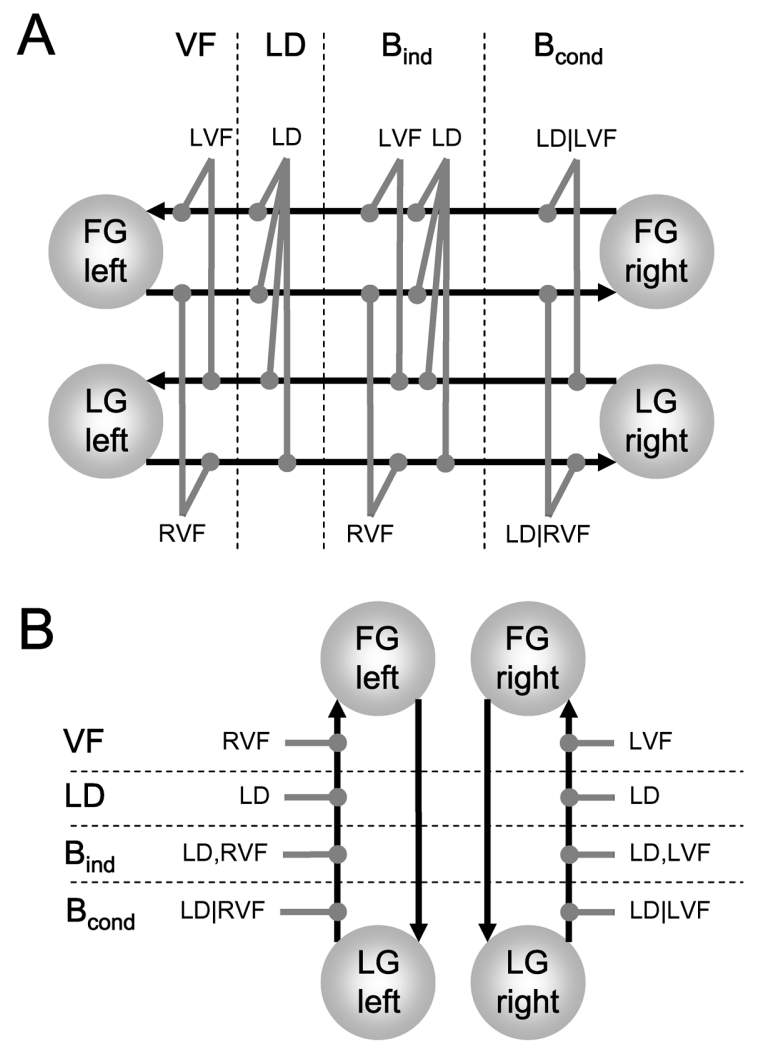

FIGURE 4. This figure describes four competing hypotheses about which experimental factors determine the strength of interhemispheric connections (A) and intrahemispheric connections (B), respectively. These different types of modulatory inputs were combined to give 16 different models (compare FIG. 3B). In contrast to the driving inputs (FIG. 3A), which were modeled as streams of events, all modulatory inputs were modeled as extended processes, that is, box-car inputs of 24-s duration.

data. This model selection problem can be addressed by means of BMS. Briefly summarized, the four competing hypotheses are the following (see FIG. 4A):

(i) Information transfer between hemispheres depends only on the visual field of stimulus presentation. This is referred to as the VF model.

(ii) Information transfer between hemispheres depends only on whether the letter decision task is performed or not. This is the LD model.

(iii) Information transfer between hemispheres depends on both the task and the visual field, but independently of each other (corresponding to a Boolean OR operation). This is the $\mathbf{B}_{\text {ind }}$ model.

(iv) Information transfer between hemispheres depends on both the task and the visual field, but in a conditional fashion: modulation of connection strength by task is only present if the stimulus was presented in a 
particular visual field (corresponding to a Boolean AND operation). This is the $\mathbf{B}_{\text {cond }}$ model.

Although less interesting in the present context, the same questions about the nature of modulatory inputs arise with respect to the intrahemispheric connections. Therefore, to perform a thorough model comparison, one needs to systematically compare all combinations of how inter- and intrahemispheric connections are changed in the four ways described above (note that, in the models presented here, we only allowed for modulation of the intrahemispheric connections from $\mathrm{LG} \rightarrow \mathrm{FG}$, but not from FG $\rightarrow$ LG; see FIG. 4B). FIgURE 3B summarizes the combinatorial logic that resulted in 16 different models that were fitted to the same data. In the following, we refer to these 16 models by first listing the modulation of the inter- and then that of the intrahemispheric connections. For example, LD-VF is the model where the callosal connections are modulated by the letter decision task and the intrahemispheric connections are modulated by the visual field.

Once the best model and thus the factors that most strongly determine transcallosal information transfer in the context of the present task are identified, we can use the posterior density of the parameters of that model to characterize mechanisms of interhemispheric interactions. For example, we can attempt to clarify at what stage of the ventral stream contextual modulation of callosal connections is present. With the exception of some EEG studies, ${ }^{40,41}$ which have rather low spatial resolution, this is a largely unexplored issue. Of even more interest, however, is whether the contextual modulation of callosal connections is asymmetric, that is, stronger for right $\rightarrow$ left connections than left $\rightarrow$ right connections or vice versa, and whether this asymmetry generalizes across the visual system or is specific to particular connections. This is a new dimension of hemispheric asymmetry that goes beyond the classical characterization of hemispheric specialization in terms of lateralized local activations and is directly related to the functional role of individual callosal "channels".

\section{RESULTS}

Here, we report the results from fitting the 16 DCMs described above to the fMRI data of a single subject from the study by Stephan et al. ${ }^{32}$ We initially present a fourarea model as shown in FIGURE 3, that is, comprising bilateral LG and FG, and subsequently extend the model to include the MOG in both hemispheres.

Starting with the four-area case, the BMS procedure indicated that, for the particular subject studied, the model that represented the best balance between model fit and model complexity was the $\mathrm{B}_{\text {cond }}-\mathrm{LD}$ model, that is, modulation of interhemispheric connections by the letter decision task conditional on the visual field of stimulus presentation and modulation of intrahemispheric connection strengths by the task only. TABLE 1 shows the Bayes factors for the comparison of the $\mathrm{B}_{\text {cond }}-\mathrm{LD}$ model with the other 15 models. The AIC and BIC approximations agreed for all comparisons. The second-best model was the LD- $\mathrm{B}_{\text {cond }}$ model (i.e., the "flipped" version of the $\mathrm{B}_{\text {cond }}-\mathrm{LD}$ model). The Bayes factor of comparing the $\mathrm{B}_{\text {cond }}-\mathrm{LD}$ with the $\mathrm{LD}-\mathrm{B}_{\text {cond }}$ model was only 2.33 , which according to the criteria summarized by Penny et al. ${ }^{23}$ (see their table 1) could be interpreted as weak evidence in favor of the $\mathrm{B}_{\text {cond }}-\mathrm{LD}$ model. All other comparisons gave Bayes factors larger than 3, representing positive, strong, or very strong evidence in favor of the $\mathrm{B}_{\text {cond }}-\mathrm{LD}$ model (see TABLE 1). 
TABLE 1. Bayes factors (BF) for comparison of the best model $\left(B_{\text {cond }^{-}}-\mathbf{L D}\right)$ with each of the other 15 models

\begin{tabular}{lrl}
\hline $\mathrm{B}_{\text {cond }}-\mathrm{LD}$ vs. & \multicolumn{1}{c}{$\mathrm{BF}$} & Evidence in favor of $\mathrm{B}_{\text {cond }}-\mathrm{LD}$ \\
\hline $\mathrm{B}_{\text {ind }}-\mathrm{B}_{\text {ind }}$ & 477.31 & Very strong \\
$\mathrm{B}_{\text {ind }}-\mathrm{B}_{\text {cond }}$ & 60.83 & Strong \\
$\mathrm{B}_{\text {ind }}-\mathrm{LD}$ & 110.84 & Strong \\
$\mathrm{B}_{\text {ind }}-\mathrm{VF}$ & 479.03 & Very strong \\
$\mathrm{B}_{\text {cond }}-\mathrm{B}_{\text {ind }}$ & 3.92 & Positive \\
$\mathrm{B}_{\text {cond }}-\mathrm{B}_{\text {cond }}$ & 4.48 & Positive \\
$\mathrm{B}_{\text {cond }}-\mathrm{VF}$ & 46267.47 & Very strong \\
LD- $\mathrm{B}_{\text {ind }}$ & 19.96 & Positive \\
LD- $\mathrm{B}_{\text {cond }}$ & 2.33 & Weak \\
LD-LD & 3.43 & Positive \\
LD-VF & 29.74 & Strong \\
VF- $\mathrm{B}_{\text {ind }}$ & 16.85 & Positive \\
VF-B cond & 4.81 & Positive \\
VF-LD & 5.59 & Positive \\
VF-VF & $1.35 \mathrm{E}+13$ & Very strong \\
\hline
\end{tabular}

Note: This table shows the Bayes factors (BF) (middle column) for the comparison of the best model $\left(\mathrm{B}_{\text {cond }}-\mathrm{LD}\right)$ with each of the other 15 models (left column). The right column lists the interpretation of the evidence in favor of the $\mathrm{B}_{\text {cond }}-\mathrm{LD}$ model according to the criteria summarized by Penny et al. ${ }^{23}$ (see their table 1).

FIGURE 5 shows the MAP estimates of the modulatory parameters ( \pm standard deviation, i.e., the square root of the posterior variances) for the $\mathrm{B}_{\text {cond }}-\mathrm{LD}$ model. The numerical values of the modulatory parameter estimates indicated an obvious hemispheric asymmetry: at the levels of both LG and FG, the MAP estimates of the modulation of the right $\rightarrow$ left connections are much larger than those of the left $\rightarrow$ right connections. However, how secure is our inference about this asymmetry? This issue can be addressed by means of contrasts of the appropriate parameter estimates. The contrasts comparing modulation of the right $\rightarrow$ left connection by $\mathrm{LD} \mid \mathrm{LVF}$ versus modulation of the left $\rightarrow$ right connection by LD $\mid \mathrm{RVF}$ are shown in FIGURE 6 (separately for connections at the levels of LG and FG, respectively). These plots indicate our certainty about asymmetrical modulation of callosal interactions through the probability that these contrasts exceed a value of zero (see eq. 6). For the particular subject shown here, we can be very certain $(98.7 \%)$ that modulation of the right $\mathrm{LG} \rightarrow$ left $\mathrm{LG}$ connection by $\operatorname{LD} \mid \operatorname{LVF}(0.34 \pm 0.14 \mathrm{~Hz})$ is larger than the modulation of the left LG $\rightarrow$ right LG connection by LD $\mid R V F(-0.08 \pm 0.16 \mathrm{~Hz})$ (compare FIGs. 4 and 5). Although there is also a clear difference in the MAP estimates of the modulatory parameters of the callosal connections at the level of the FG, the difference is smaller and the variance of these estimates is larger $(0.13 \pm 0.19 \mathrm{~Hz} \mathrm{vs}$. $0.01 \pm 0.17 \mathrm{~Hz}$ ). Consequently, for the specific subject studied, we have little confidence $(68.0 \%)$ that the asymmetry observed for the LG connections also exists for callosal connections between right and left FG. Similarly, even though the MAP estimates indicated that the modulation of the intrahemispheric $\mathrm{LG} \rightarrow \mathrm{FG}$ connection by task demands was larger in the left hemisphere $(0.44 \pm 0.14 \mathrm{~Hz})$ than in the right 


\section{$\mathrm{B}_{\text {cond }^{-}}$LD model (4 areas)}

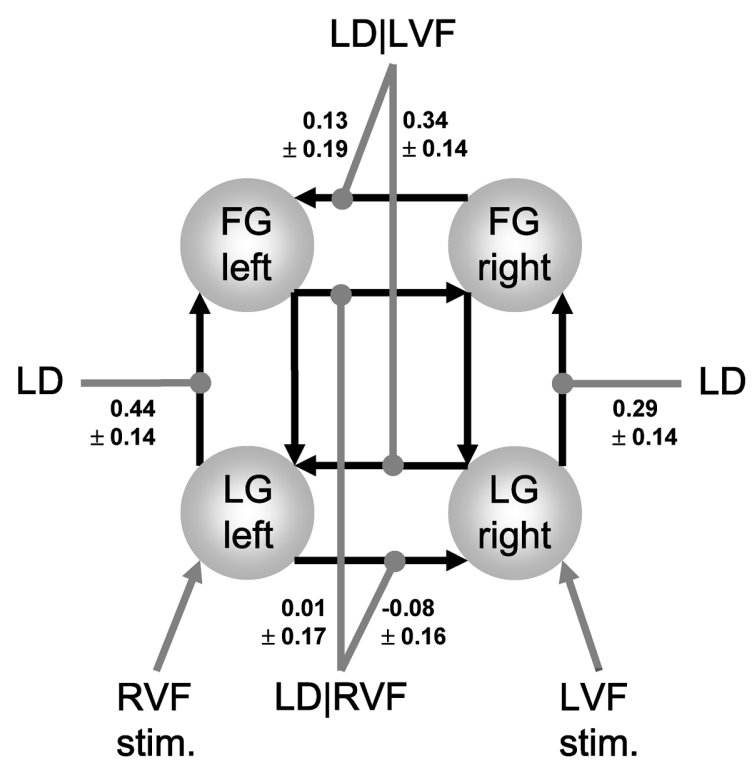

FIGURE 5. This figure shows the maximum a posteriori (MAP) estimates of the parameters ( \pm square root of the posterior variances; units: $1 / \mathrm{s}=\mathrm{Hz}$ ) for the $\mathrm{B}_{\text {cond }}-\mathrm{LD}$ model, which for the particular subject studied proved to be the best of all 16 models tested. For clarity, only the parameters of interest, that is, the modulatory parameters of inter- and intrahemispheric connections, are shown and the bilateral visual field input has been omitted.

hemisphere $(0.29 \pm 0.14 \mathrm{~Hz})$, we only have a very modest certainty $(75.2 \%)$ about the presence of this form of connectional asymmetry.

Since the difference between the best and the second-best model was not huge (see above), we also investigated the parameter estimates for the LD-B $\mathrm{B}_{\text {cond }}$ model. In this model, task demands alone, independent of visual field, determined the strength of callosal connections. It was pleasing to find that this model gave compatible results in terms of the asymmetrical modulation of callosal connections. Here, there was a $95.6 \%$ confidence that modulation of the right LG $\rightarrow$ left LG connection by $\mathrm{LD}(0.43 \pm 0.15 \mathrm{~Hz})$ was larger than the equivalent modulation of the left LG $\rightarrow$ right LG connection by LD $(0.03 \pm 0.16 \mathrm{~Hz})$. As with the $\mathrm{B}_{\text {cond }}-\mathrm{LD}$ model, we were considerably less certain (67.7\%) about this asymmetry for the callosal connections at the level of FG $(0.31 \pm 0.18 \mathrm{~Hz}$ vs. $0.19 \pm 0.17 \mathrm{~Hz})$.

Finally, we extended the $\mathrm{B}_{\text {cond }}-\mathrm{LD}$ model to include left and right MOG. Fitting this six-area model to the data gave estimates that were only marginally different for the modulatory parameters of LG and FG connections discussed above (see FIG. 7). In contrast, the MAP estimates for modulation of the callosal connections between left and right $\mathrm{MOG}$ were very close to zero (modulation of the right MOG $\rightarrow$ left MOG 
A

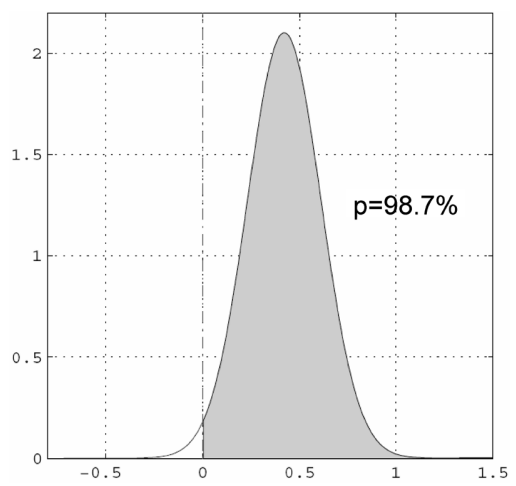

Contrast:

modulation of right LG $\rightarrow$ left LG by LD|LVF versus

modulation of left LG $\rightarrow$ right LG by LD|RVF
B

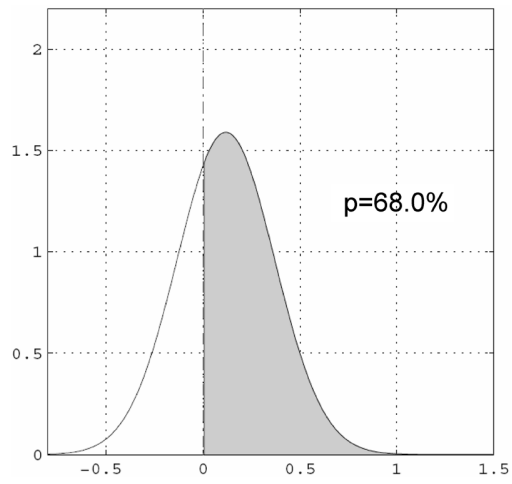

Contrast:

modulation of right FG $\rightarrow$ left FG by LD|LVF versus

modulation of left FG $\rightarrow$ right FG by LD|RVF

FIGURE 6. Asymmetry of callosal connections with regard to contextual modulation. The plots show the probability that the modulation of the right $\rightarrow$ left connection by task conditional on left visual field stimulation is stronger than the modulation of the left $\rightarrow$ right connection by task conditional on right visual field stimulation. (A) For the callosal connections between left and right LG, we can be very confident that this asymmetry exists: the probability of the contrast being larger than zero is $p\left(c^{T} \eta_{\theta \mid y}>0\right)=98.7 \%$. (B) For the callosal connections between left and right FG, we are considerably less certain about the presence of this asymmetry: the probability of the contrast being larger than zero is $p\left(c^{T} \eta_{\theta \mid y}>0\right)=68.0 \%$.

connection by LD|LVF: $0.01 \pm 0.02 \mathrm{~Hz}$; modulation of the left MOG $\rightarrow$ right MOG connection by LD|RVF: $-0.01 \pm 0.02 \mathrm{~Hz}$ ). Given these very small effects, the contrast between these estimates unsurprisingly indicated that there was no support for the presence of an asymmetry with regard to contextual modulation of callosal connections between left and right MOG: the probability $p\left(c^{T} \eta_{\theta \mid y}>\gamma\right)=54.2 \%$ was very close to the $50 \%$ margin, indicating that it is equally likely that $c^{T} \eta_{\theta \mid y}$ is smaller or larger than zero.

\section{DISCUSSION}

In this article, we have summarized the theoretical foundations of DCM and BMS and have provided an example of the practical application of these techniques, using data from a single subject in the study by Stephan et al.$^{32}$ DCM enables the investigation of how neural systems (composed of large neural populations like cortical areas) that have relatively high connectional complexity, for example, multiple reciprocal connections and loops, ${ }^{11,23}$ operate. Together with BMS, DCM is a powerful tool to clarify which of multiple experimental manipulations (e.g., stimulus type, induction 


\section{$\mathrm{B}_{\text {cond }^{-}}$LD model (6 areas)}

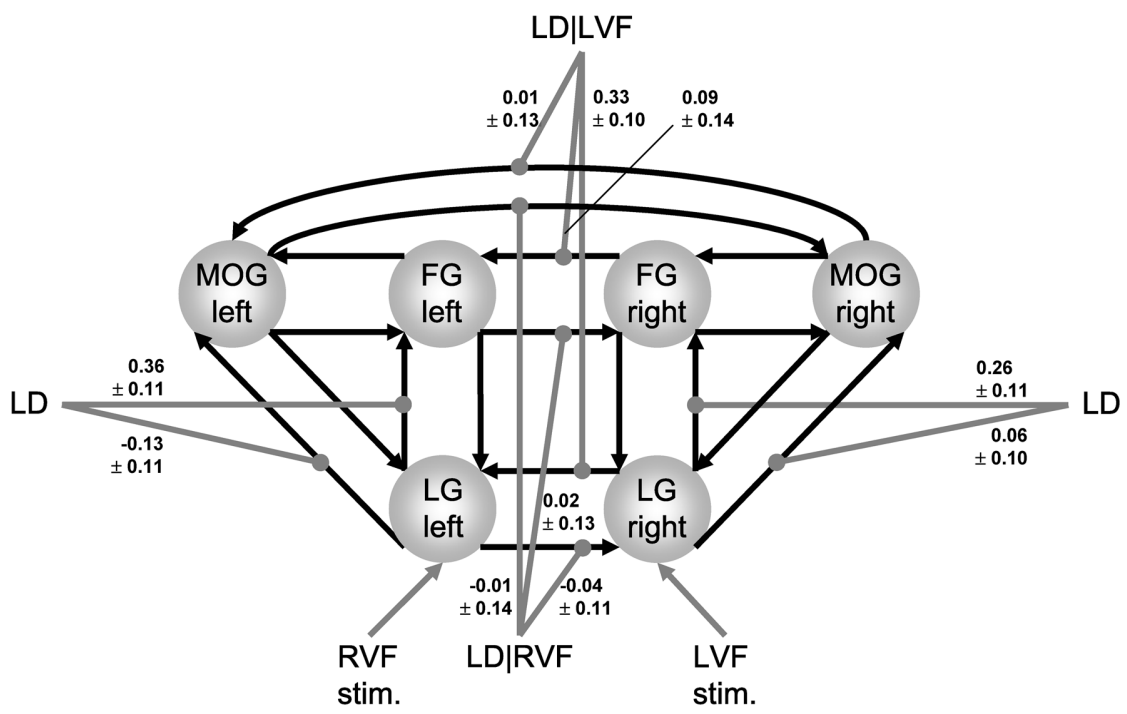

FIGURE 7. Parameter estimates for an extended version of the $\mathrm{B}_{\text {cond }}-\mathrm{LD}$ model that includes the MOG in both hemispheres (see FIG. 5 for details). The estimates for callosal connections at the level of LG and FG were very similar to those from the four-area model (FIG. 5). In contrast to LG, there was no evidence for contextual modulation of callosal connections between right and left MOG.

of cognitive set, learning processes, etc.) have a significant impact on the dynamics of the network under investigation. By representing experimental factors as external inputs in the model, modeled effects can be interpreted fairly directly in neurobiological terms: any given DCM specifies precisely where inputs enter and whether they are driving (i.e., exert their effects through direct synaptic responses in the target area) or modulatory (i.e., exert their effects through changing synaptic responses in the target area to inputs from another area). This distinction, made at the level of neural populations, has a nice correspondence to empirical observations that single neurons can have either driving or modulatory effects on other neurons. ${ }^{23}$

There are several ways in which DCM and DWI techniques can profit from each other. We have given a simple example in this paper of how models of effective connectivity like DCM are important for our understanding of processes in neural systems, even if their anatomical connectivity is well understood. On the other hand, there are obviously many systems whose connectivity is less well known than that of the corpus callosum and visual areas. In this case, the specification of DCMs could be greatly facilitated by precise anatomical data on human brain connectivity obtained by DWI. Another potential opportunity is to use the likelihood of the 
existence of particular connections (as obtained from probabilistic tractography methods ${ }^{7,8}$ ) as priors on connection strengths in Bayesian models like DCM.

In our empirical example, we demonstrated two things: in the particular individual studied, there is good evidence that (i) the measured data are best explained by a model in which interhemispheric interactions depend on task demands, but conditional on the visual field of stimulus presentation, and (ii) there is a hemispheric asymmetry in context-dependent transcallosal interactions. Importantly, this asymmetry was not equally pronounced for all visual areas studied. It was particularly strong for the callosal connections between left and right LG: performance of the LD task specifically enhanced the strength of the influence of the right on the left LG, but only if the stimulus was presented in the left visual field, and thus the information was initially only available in the right hemisphere. The reversed conditional effect, that is, modulation of left LG $\rightarrow$ right LG by LD|RVF, was much weaker (and actually slightly negative; see FIGS. 5 and 7). This result means that, for the particular paradigm used, enhancement of callosal connections was only necessary if stimulus information was initially represented in the "suboptimal", that is, right, hemisphere. Interestingly, in the subject analyzed, this asymmetry was less pronounced at the level of the FG and virtually absent at the level of the MOG.

These results complement very nicely our previous results on local activations elicited by this paradigm. ${ }^{32}$ While we had previously established, using identical stimuli in all conditions, that a change in task demands was sufficient to determine the lateralization of brain activity, our previous analyses were not suited to clarify the principles according to which the two hemispheres functionally interacted. In other words, despite empirical data on context-dependent activations of visual areas and good knowledge about the general anatomical connectivity between these areas, we could not infer the mechanism by which transcallosal interactions contributed to the observed activations. In this paper, we have shown how this can be achieved by comparing different models, which are fitted to the empirical data, and then making statistical inferences about the parameters of the optimal model. It should be emphasized, however, that in this paper we have applied DCM to data from a single subject only, and it remains to be seen whether or not the principles of interhemispheric interactions found for the particular individual studied here generalize to the population. To clarify this question, we are currently analyzing the data from all subjects of the study by Stephan et al. ${ }^{32}$ following the strategy outlined in this article.

Group studies with DCM and BMS require somewhat different statistical procedures from those used in this paper. For example, when trying to find the optimal model for a group of individuals by BMS, it is likely that the optimal model will vary, at least to some degree, across subjects. An overall decision for $n$ subjects can be made by computing an average Bayes factor, which corresponds to the $n$-th root of the product of the individual Bayes factors (note that multiplication is appropriate because model comparisons from different individuals are statistically independent). Moreover, in this paper, all inferences about coupling parameters were based on the magnitude of the effect of interest (i.e., the contrast of the appropriate MAP estimates) compared to the precision with which this estimate was obtained (i.e., the posterior variance). When dealing with a group of subjects, one may be interested in different types of inference. For example, one may want to establish that a certain effect (e.g., the modulation of a particular connection by some experimental condition) is consistently expressed across subjects. This second-level inference can take various forms. 
For example, one can apply a classical statistical test to the parameters of interest or one can use a Bayesian approach, either treating the individual effects as fixed and combining the posterior variances or treating the individual parameters as random effects, thus enabling inference beyond the particular group of subjects studied. ${ }^{42}$ These topics will be the subject of forthcoming methodological papers on DCM.

It should be noted that the idea underlying dynamic causal models is not restricted to fMRI data. David and Friston ${ }^{43}$ have recently developed a neural mass model that, when combined with an appropriate forward model and estimation scheme, can be used as a DCM to derive neural coupling parameters from empirically measured electroencephalographic (EEG) or magnetoencephalographic (MEG) data (see David et al. ${ }^{44}$ for a first application). This model has a much more sophisticated neural state equation than equation 2 in this paper, distinguishing between different cortical layers and different neural populations with specific time constants and connectivity. Furthermore, Harrison et al. ${ }^{45}$ have developed a mean field model for event-related potentials that uses stochastic differential equations for the description of neural processes involving different types of transmitter receptors. The coming years will see a further refinement of dynamic causal models of this kind.

We conclude by emphasizing that one of the long-term goals of developments related to DCM is to obtain tools for clinical applications. Such tools are particularly important for the study of psychiatric diseases like schizophrenia whose phenotypes are often confusingly heterogeneous due to strong interactions between genotype and environmental influences. One aim is to determine disease-specific endophenotypes; these are biological markers at intermediate levels between genome and behavior, for example, particular neurophysiological or neurochemical indices. ${ }^{46}$ For example, if the pathophysiological mechanism that underlies a specific disease is an abnormal functional coupling between two or more brain regions in a particular context, this would correspond to a disease-specific pattern of coupling parameters in an appropriate DCM. The challenge for the future will be to establish models of valid neural systems that can be fitted to measured imaging data and that are sensitive enough that their connectivity parameters can be used reliably for the diagnostic classification of individual patients. Ideally, such models should be used in conjunction with "implicit" paradigms that are minimally dependent on patient compliance, for example, mismatch negativity paradigms. ${ }^{47}$ Given established validity and sufficient sensitivity of such a model, one could use it by analogy to a biochemical laboratory test, that is, to compare a particular model parameter (or combinations thereof) against reference distributions in order to obtain diagnostic classifications. If the model is sophisticated enough to distinguish between different transmitter receptors, it might also be possible to obtain predictions for the optimal pharmacological treatment of individual patients. DCM, as described in this paper, provides a generic framework and starting point for this long-term endeavor.

\section{ACKNOWLEDGMENTS}

This work was supported by the Wellcome Trust (to K. E. Stephan, W. D. Penny, and K. J. Friston), the Medical Research Council (to J. C. Marshall), and the Deutsche Forschungsgemeinschaft (to G. R. Fink). 


\section{REFERENCES}

1. FRISTON, K.J. 2002. Beyond phrenology: what can neuroimaging tell us about distributed circuitry? Annu. Rev. Neurosci. 25: 221-250.

2. Marshall, J.C. \& G.R. FinK. 2003. Cerebral localization, then and now. NeuroImage 20(suppl. 1): S2-S7.

3. Köвbert, C., R. Apps, I. Bechmann et al. 2000. Current concepts in neuroanatomical tracing. Prog. Neurobiol. 62: 327-351.

4. Stephan, K.E., L. Kamper, A. Bozkurt et al. 2001. Advanced database methodology for the collation of connectivity data on the macaque brain (CoCoMac). Philos. Trans. R. Soc. Lond. B Biol. Sci. 356: 1159-1186.

5. Bürgel, U., T. Schormann, A. SChleicher et al. 1999. Mapping of histologically identified long fiber tracts in human cerebral hemispheres to the MRI volume of a reference brain: position and spatial variability of the optic radiation. NeuroImage 10: $489-499$.

6. Galuske, R.A., W. Schlote, H. Bratzke et al. 2000. Interhemispheric asymmetries of the modular structure in human temporal cortex. Science 289: 1946-1949.

7. Behrens, T.E.J., M.W. Woolrich, M. Jenkinson et al. 2003. Characterization and propagation of uncertainty in diffusion-weighted MR imaging. Magn. Reson. Med. 50: $1077-1088$.

8. Parker, G.J.M., H.A. Haroon \& C.A.M. Wheeler-Kingshott. 2003. A framework for a streamline-based probabilistic index of connectivity (PICo) using a structural interpretation of MRI diffusion measurements. J. Magn. Reson. Imag. 18: 242-254.

9. Alexander, D.C., G.J. Barker \& S.R. Arridge. 2002. Detection and modeling of non-Gaussian apparent diffusion coefficient profiles in human brain data. Magn. Reson. Med. 48: 331-340.

10. Strogatz, S.H. 2001. Exploring complex networks. Nature 410: 268-276.

11. Friston, K.J., L. Harrison \& W. PenNY. 2003. Dynamic causal modelling. NeuroImage 19: $1273-1302$.

12. Rockland, K.S. \& D.N. Pandya. 1979. Laminar origins and terminations of cortical connections of the occipital lobe in the rhesus monkey. Brain Res. 179: 3-20.

13. Ringo, J.L., R.W. Doty, S. Demeter et al. 1994. Time is of the essence: a conjecture that hemispheric specialization arises from interhemispheric conduction delay. Cereb. Cortex 4: 331-343.

14. CAVAda, C. \& P.S. Goldman-Rakic. 1989. Posterior parietal cortex in rhesus monkey: I. Parcellation of areas based on distinctive limbic and sensory corticocortical connections. J. Comp. Neurol. 287: 393-421.

15. McGuiRE, P.K., J.F. BATES \& P.S. GoldMAN-RAKIC. 1991. Interhemispheric integration: I. Symmetry and convergence of the corticocortical connections of the left and the right principal sulcus (PS) and the left and the right supplementary motor area (SMA) in the rhesus monkey. Cereb. Cortex 1: 390-407.

16. Funnell, M.G., P.M. Corballis \& M.S. GazZaniga. 2000. Insights into the functional specificity of the human corpus callosum. Brain 123: 920-926.

17. Westerhausen, R., F. Kreuder, S. Sequeira et al. 2004. Effects of handedness and gender on macro- and microstructure of the corpus callosum and its subregions: a combined high-resolution and diffusion-tensor MRI study. Cogn. Brain Res. 21: 418-426.

18. Hulshoff Pol, H.E., H.G. SchnACK, R.C. Mandl et al. 2003. Focal white matter density changes in schizophrenia: reduced inter-hemispheric connectivity. NeuroImage 21: $27-35$.

19. BANICH, M.T. 1998. The missing link: the role of interhemispheric interaction in attentional processing. Brain Cogn. 36: 128-157.

20. Bollen, K.A. 1989. Structural Equations with Latent Variables. Wiley. New York.

21. Kennedy, H., C. Dehay \& J. Bullier. 1986. Organization of the callosal connections of visual areas V1 and V2 in the macaque monkey. J. Comp. Neurol. 247: 398-415.

22. Segraves, M.A. \& A.C. Rosenquist. 1982. The afferent and efferent callosal connections of retinotopically defined areas in cat cortex. J. Neurosci. 2: 1090-1107.

23. Penny, W.D., K.E. Stephan, A. Mechelli et al. 2004. Comparing dynamic causal models. NeuroImage 22: 1157-1172. 
24. Sherman, S.M. \& R.W. Guillery. 1998. On the actions that one nerve cell can have on another: distinguishing "drivers" from "modulators". Proc. Natl. Acad. Sci. USA 95: 7121-7126.

25. Buxton, R.B., E.C. Wong \& L.R. FRANK. 1998. Dynamics of blood flow and oxygenation changes during brain activation: the balloon model. Magn. Reson. Med. 39: 855-864.

26. Friston, K.J., A. Mechelli, R. Turner et al. 2000. Nonlinear responses in fMRI: the balloon model, Volterra kernels, and other hemodynamics. NeuroImage 12: 466-477.

27. Friston, K.J. 2002. Bayesian estimation of dynamical systems: an application to fMRI. NeuroImage 16: 513-530.

28. Penny, W.D., K.E. Stephan, A. Mechelli et al. 2004. Modelling functional integration: a comparison of structural equation and dynamic causal models. NeuroImage 23: S264-S274.

29. Pitt, M.A. \& I.J. Myung. 2002. When a good fit can be bad. Trends Cogn. Sci. 6: 421-425.

30. StePhAN, K.E. 2004. On the role of general systems theory for functional neuroimaging. J. Anat. 205: 443-470.

31. McIntosh, A.R., C.L. Grady, L.G. Ungerleider et al. 1994. Network analysis of cortical visual pathways mapped with PET. J. Neurosci. 14: 655-666.

32. StePhAN, K.E., J.C. MARShAll, K.J. FRISTON et al. 2003. Lateralized cognitive processes and lateralized task control in the human brain. Science 301: 384-386.

33. JenNer, A.R., G.D. Rosen \& A.M. GalabuRDA. 1999. Neuronal asymmetries in primary visual cortex of dyslexic and nondyslexic brains. Ann. Neurol. 46: 189-196.

34. Amunts, K., A. Schleicher, U. Bürgel et al. 1999. Broca's region revisited: cytoarchitecture and intersubject variability. J. Comp. Neurol. 412: 319-341.

35. Sergent, J. 1983. Role of the input in visual hemispheric asymmetries. Psychol. Bull. 93: $481-512$.

36. Levy, J. \& C. Trevarthen. 1976. Metacontrol of hemispheric function in human splitbrain patients. J. Exp. Psychol. Hum. Percept. Perform. 2: 299-312.

37. Fink, G.R., P.W. Halligan, J.C. Marshall et al. 1996. Where in the brain does visual attention select the forest and the trees? Nature 382: 626-628.

38. Abel, P.L., B.J. O'Brien \& J.F. OlavarRia. 2000. Organization of callosal linkages in visual area V2 of macaque monkey. J. Comp. Neurol. 428: 278-293.

39. KÖtTER, R. \& K.E. STEPHAN. 2003. Network participation indices: characterizing component roles for information processing in neural networks. Neural Networks 16: $1261-1275$.

40. SChack, B., S. Weiss \& P. Rappelsberger. 2003. Cerebral information transfer during word processing: where and when does it occur and how fast is it? Hum. Brain Map. 19: $18-36$.

41. Nowicka, A., A. GrabowsKa \& E. Fersten. 1996. Interhemispheric transmission of information and functional asymmetry of the human brain. Neuropsychologia 34: 147-151.

42. Penny, W. \& A.P. Holmes. 2004. Random-effects analysis. In Human Brain Function, pp. 843-850. Elsevier. Amsterdam/New York/San Diego.

43. DAVID, O. \& K.J. FrISTON. 2003. A neural mass model for MEG/EEG: coupling and neuronal dynamics. NeuroImage 20: 1743-1755.

44. David, O., L. Harrison, J. KiLner et al. 2004. Studying effective connectivity with a neural mass model of evoked MEG/EEG responses. In Proceedings of the 14th International Conference on Biomagnetism (BIOMAG 2004), pp. 135-138. Boston.

45. Harrison, L.M., O. David \& K.J. Friston. 2005. Stochastic models of neuronal dynamics. Philos. Trans. R. Soc. Lond. B Biol. Sci. 360: 1075-1091.

46. GotTesman, I.I. \& T.D. Gould. 2003. The endophenotype concept in psychiatry: etymology and strategic intentions. Am. J. Psychiatry 160: 636-645.

47. Baldeweg, T., A. Klugman, J. Gruzelier et al. 2004. Mismatch negativity potentials and cognitive impairment in schizophrenia. Schizophr. Res. 69: 203-217. 